\title{
An Approach to Reduction of Hysteresis in Smart Materials
}

\author{
Juan Manuel Cruz-Hernández and Vincent Hayward \\ Department of Electrical Engineering and Center for Intelligent Machines \\ McGill University \\ 3480 University Street, Montréal, Qc, H3A 2A7, Canada \\ http://www.cim.mcgill.ca/ $\{$ manuel, hayward $\} /$ Home.html
}

\begin{abstract}
This paper addresses the problem of reducing the hysteresis found in the actuation of most smart materials. They are divided in two groups: systems with no saturation (e.g. piezoelectric actuators), and systems with saturation (e.g. Shape Memory Actuators). For the control of the first group the concept of phaser is introduced, an operator which shifts the phase of a periodic signal but keeps its magnitude unchanged. Since it is possible to approximate phasers with linear filters, it is possible to design practical compensators. The design of a phaser requires the knowledge of one parameter $\phi$, easily identified from experimental transfer function estimates. For the second group, two phasers are used in a tandem connection. One phaser is designed as described before, and the second is designed so as to vary with the input. This compensation reduces the hysteresis to a single saturation. To show its effectiveness, simulation results are provided using the hystery model, then the method is applied to an SMA actuator. The effectiveness of a single phaser for non-saturated hysteresis have already been experimentally demonstrated in previous work.
\end{abstract}

\section{Introduction}

Hysteresis is found in most actuation systems. With reference to smart materials, hysteresis is significant in two commonly used materials: piezoelectric ceramics and shape memory alloys. In a restricted frequency range, it is possible to consider that, in these materials, hysteresis is rate independent and acts as an additive disturbance on the linear dynamics of the system. Here, a system with hysteresis is seen as a parallel connection of a linear dynamical system with a rate independent hysteresis with memory. In operator form the system can be represented by [2]:

$$
y=\mathbf{L}[u]+\hat{\boldsymbol{\Gamma}}[u]
$$

where $\hat{\boldsymbol{\Gamma}}$ represents the rate independent hysteresis with memory and $\mathbf{L}$ represents the dynamics of the system. This may also be viewed as the two first terms of a Volterra series expansion.

\subsection{Modeling and Control of Hysteresis}

Modeling of hysteresis in smart materials has been studied by Hughes and Wen in $[7,8]$, where the Preisach model [10] is used to model the static behavior of a flexible beam, which uses two piezoelectric actuators and a shape memory alloy. Experiments are used to show that the Preisach model can be used to model their system. In [3], Ge and Jouaneh model hysteresis found in a piezoelectric actuator using the Preisach model. Goldfarb and Celanovic [4], introduced a causal representation of rate independent hysteresis found in a piezoelectric stack actuator, incorporating a generalized Maxwell resistive capacitor as a lumped parameter. For more theoretical models of hysteresis, the reader is referred to Visintin [13], Mayergoyz [10], Krasnoselskii and Pokrovskii [9] or Brokate et al [1].

Control of systems with hysteresis has been considered by Ge and Jouaneh [3], where a combination of a feedforward loop (including a nonlinearity) with a feedback loop (PID) is used. Tao and Kokotović [11] have developed control algorithms to reduce the effects of nonlinearities like hysteresis in a plant represented by a linear part preceded by a hysteresis characteristic. In [5], Gorbet and Wang gave passivity conditions for a PI control to remain stable.

\subsection{Approach}

The compensation of hysteresis is considered for periodic signals in a given frequency range. In most cases, this frequency range will correspond simply to the practical bandwidth of a particular actuator. The effect of hysteresis is considered to be a combination of phase distortion and magnitude distortion. The present approach considers compensators which can correct for the phase distortion while ignoring the magnitude distortion. When successful, the compensation results in a singular nonlinearity which can be corrected separately if needed. In [2] this approach 
was applied experimentally to the correction of non saturating hysteresis in a piezoelectric actuator. The present paper extends that approach to reduce hysteresis $\hat{\boldsymbol{\Gamma}}[u]$, which can have saturation as in SMA actuators.

The reduction of hysteresis with saturation is achieved by applying phase correction by successive approximations using a two stage controller.

In section 2 , the hystery unit model is recalled. In section 3, a brief introduction to the phaser approach to the correction of hysteresis is given. In section 4 , systems which present hysteresis with no saturation are considered. The reduction of this type of hysteresis is done using a variation of the phaser presented in [2]. In section 5, the phaser is used to implement what will be called a variable phaser which reduces the hysteresis with saturation into a single nonlinearity, by making the phase correction depend on the magnitude of the input. Section 6 presents simulations results while reducing a hysteresis with saturation in the hystery model, and experimental results using an SMA actuator. Finally, in section 7, conclusions and future work are summarized.

\section{The hystery unit model}

The hystery unit is discussed in [12]. The upper and lower branches of the hysteresis loop are both segments of hyperbolic tangent functions, and they are described as a two families of curves. The placement of these segments along the $\mathrm{x}$-axis is $H_{c} \geq 0$.

The set of rising curves that passes through all possible starting points forms the family of rising curves. Each member, indexed by the number $\eta_{k}^{+}$, has the form:

$$
\begin{aligned}
y_{k}^{+}=\eta_{k}^{+}+\left(1-\eta_{k}^{+}\right) \tanh \left(x_{k}^{+}-H_{c}\right) ; \\
\text { where } \\
\eta_{k}^{+}=\frac{y_{k-1}^{-}-\tanh \left(x_{k-1}^{-}-H_{c}\right)}{1-\tanh \left(x_{k-1}^{-}-H_{c}\right)}
\end{aligned}
$$

The family of falling curves, indexed by the number $\eta_{k}^{-}$, has the form

$$
\begin{aligned}
& \qquad y_{k}^{-}=-\eta_{k}^{-}+\left(1-\eta_{k}^{-}\right) \tanh \left(x_{k}^{-}+H_{c}\right) ; \\
& \text { where } \quad \eta_{k}^{-}=\frac{y_{k}^{+}-\tanh \left(x_{k}^{+}+H_{c}\right)}{-1-\tanh \left(x_{k}^{+}+H_{c}\right)}
\end{aligned}
$$

This model has a full memory of the history of inputs and can approximate the hysteresis found in magnetization. The hystery model is one of the simplest among all hysteresis models. It is much simpler than the Preisach model which requires experimental data to be conveniently implemented in a computer.

\section{The phaser}

In [2], the effect of hysteresis is seen as a phase shift between the input to the system and its output, therefore, only periodic signals are considered. An elementary operator termed the phaser $\left(\mathbf{L}_{p a}\right)$ shifts its periodic input signal by a constant angle $\phi>0$, with a constant magnitude of 1 , independent from the frequency or the magnitude of the input signal.

The frequency domain representation of the phaser is an imaginary number:

$$
\begin{gathered}
\mathbf{L}_{p a}(j \omega)=a+j b \\
\left|\mathbf{L}_{p a}(j \omega)\right|=1 \quad \& \quad \varangle \mathbf{L}_{p a}(j \omega)=\phi \\
a=\frac{\tan \phi}{\sqrt{1+\tan ^{2} \phi}} \quad \& \quad b=\frac{1}{\sqrt{1+\tan ^{2} \phi}}
\end{gathered}
$$

It is impossible to realize a causal system that shifts the input by a constant angle over the whole frequency range. In practice, an approximation to the phaser is taken using a series of lead controllers (see Figure 1). This controllers shift the input signal over a finite frequency range, which is chosen to be the bandwidth of the system (usually low frequencies). When the shift angle is small, the phase and magnitude error are quite small. For example, for a shift of 5 degrees and a phase error smaller than a tenth of a degree, the magnitude error is about $1 \mathrm{~dB}$ per decade covered.

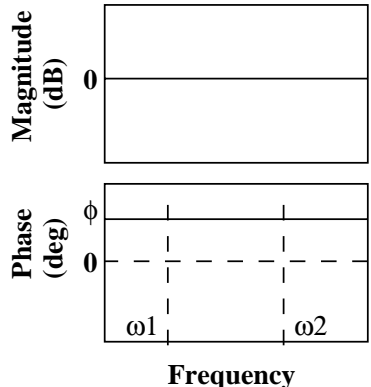

a)

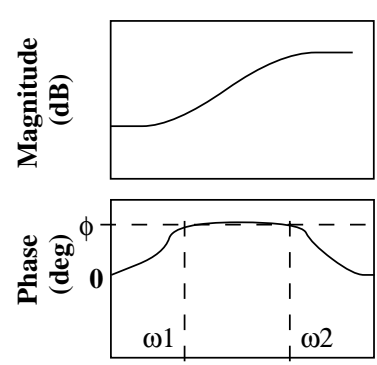

Frequency
Figure 1: Frequency response: a) An ideal phaser. b) An approximation to the phaser over a certain frequency range.

In the design of a practical compensator, $\phi$ is the only parameter to be determined. One way to obtain $\phi$ is to produce an open loop Bode plot of the system (specifically the phase plot at the low frequency range, where the phase is never 0 or a multiple of $90^{\circ}$ ). This method was experimentally applied to a piezoelectric actuator [2]. 


\section{Reduction of Hysteresis with no sat- uration}

Equation (4) can be rewritten as:

$$
\mathbf{L}_{p a}(j \omega)=\cos (\phi)+\frac{\sin (\phi)}{\omega} s
$$

where in this case $\cos (\phi)=a, \sin (\phi)=b$ and $s=j \omega$, or in the time domain:

$$
u(t)=\cos (\phi) u(x)+\frac{\sin (\phi)}{\omega} \dot{u}(t)
$$

where $y(t)$ is the output, $\phi$ is the phase lag between the input and the output, $\omega$ is the frequency of the input signal, and $u(t)$ is the input signal.

Consider Figure 2 which shows a typical response of a system with no saturation. If the phase lag between input an output is measured ${ }^{1}$, the angle $\phi$ required for the design of the phaser is immediately obtained. The hysteresis loop is viewed as a phaser with a negative angle.

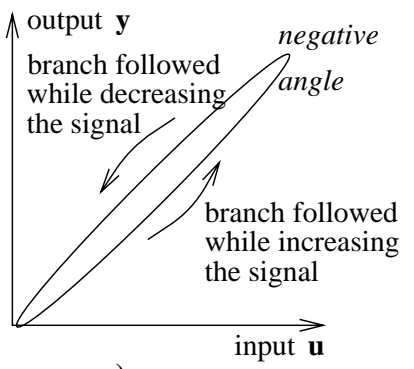

a)

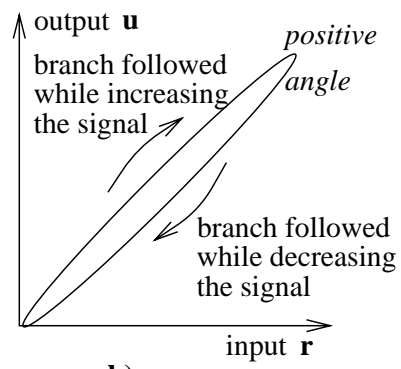

b)
Figure 2: a) Typical hysteresis loop with no saturation, with a phase angle $-\phi$. b) The phaser loop with an angle $\phi$.

If this phaser is connected in cascade with a hysteresis with no saturation, a perfect compensation is obtained because one block cancels the other as in Figure 3.

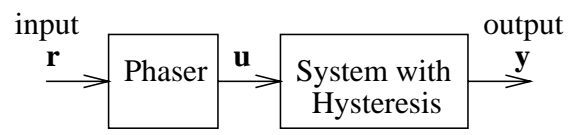

Figure 3: The phaser in cascade with a plant without hysteresis.

\footnotetext{
${ }^{1}$ One other method is to approximate the hysteresis loop by the set of coordinates $(\sin (x), \sin (x-\phi))$, where $x=\{x \mid x \in[0,2 \pi]\}$, and $\phi$ is the angle which will be varied until the hysteresis loop is best approximated.
}

\section{Hysteresis with saturation}

Consider the hysteresis in Figure 4.a). If the phaser described in the previous section is used, the result must be imperfect: depending on the input amplitude, there must be over- or under-compensation, see Figure 4.c) for the result of the compensation of Figure 4.a).

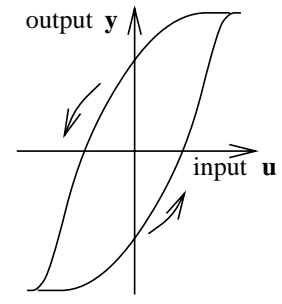

a)

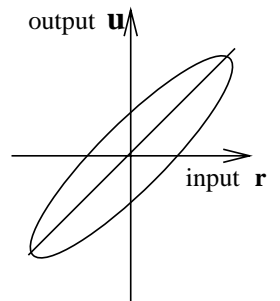

b)

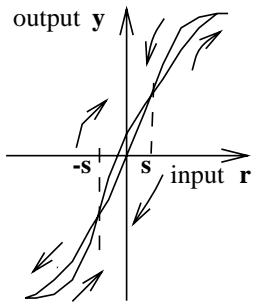

c)
Figure 4: a) Hysteresis loop with saturation. b) Phaser loop. c) Loop after using a phaser in b) with the hysteresis loop of a).

The effect of saturation is to cause imperfect compensation leading to an input-output phase plot having possibly several loops. The loop in the middle resembles a hysteresis loop with no saturation. The loops at the ends of the graph are symmetric and have a positive phase angle. The second approximation involves a second phaser to provide a positive angle $\phi_{1}$ for the middle loop, and a negative angle $\phi_{2}$ for the others. These angles, once again, can be obtained considering each loop independent of the others, measured as before.

The control strategy for hysteresis with saturation will be now as shown in Figure 5 .

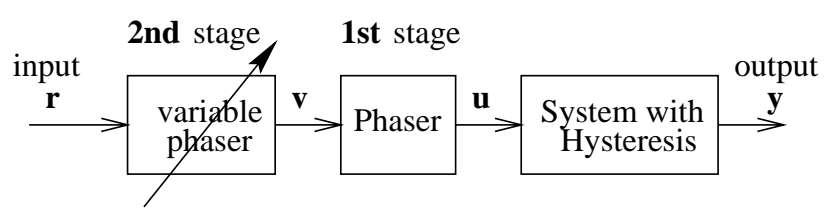

Figure 5: Controller diagram.

The second stage is a variable phaser since its phase angle varies from the positive angle $\phi_{1}$ to the negative angle $\phi_{2}$. Therefore, its mathematical expression is:

$v(t)=\left\{\begin{array}{lll}\cos \left(\phi_{1}\right) r(t)+\frac{\sin \left(\phi_{1}\right)}{\omega} \dot{r}(t) & \text { if } & |r| \leq s \\ \cos \left(\phi_{2}\right) r(t)+\frac{\sin \left(\phi_{2}\right)}{\omega} \dot{r}(t) & \text { if } & |r|>s\end{array}\right.$

where $\phi_{1} \geq 0$ and $\phi_{2} \leq 0$, and $s$ is a suitable value obtained from Figure 4.c) where a change in the orientation of the loop occurs. The phase will be changing as in Figure 6, as opposed to the constant phase of the original phaser. 


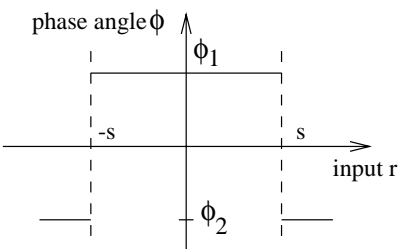

Figure 6: Variation of the phase angle as a function of the input.

\section{Simulations and experimental re- sults}

The simulated hysteresis loop, using the hystery model, is shown in Figure 7.

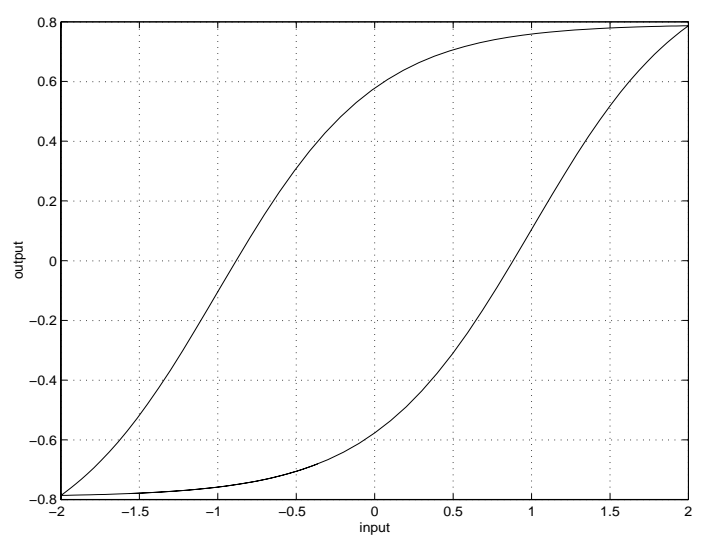

Figure 7: Hysteresis loop.

The result after using the first stage of the controller in Figure 5, with $\phi=25$ deg., is shown in Figure 8.

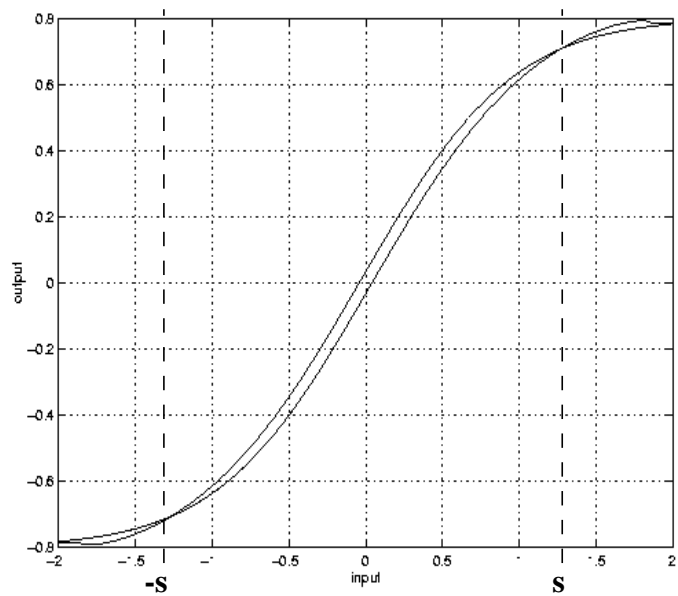

Figure 8: Input-output response after a first stage controller (phaser) was used.

The first stage gives as a result a reduced area inside the loop, but the original loop is divided in three, as predicted before. The loop created in the middle of the graph is a loop with negative phase and the loops at the ends have positive phase, as predicted.

A second phaser is designed using equation (7) with values: $\phi_{1}=1.25 \mathrm{deg} ., \phi_{2}=-1.64 \mathrm{deg}$. , and $s=-1.39$. The result of the two stages controller, is presented in Figure 9. The second controller yields

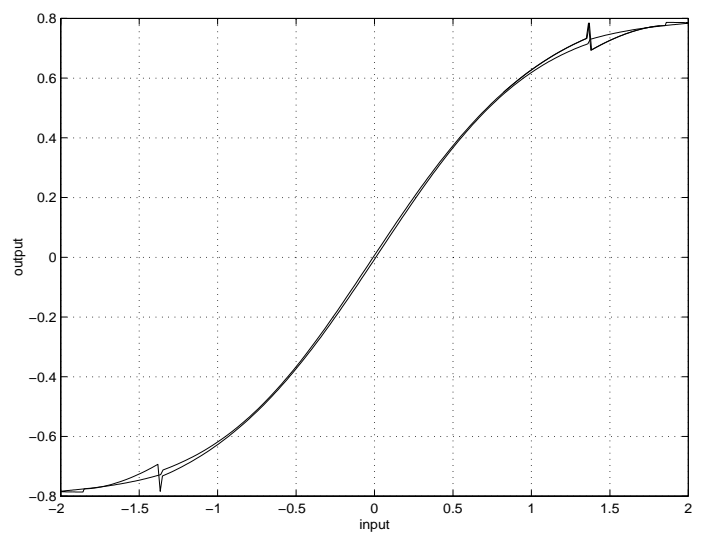

Figure 9: Input-output response after a two stage controllers were used. The second stage is a phaser varying with discrete values of $\phi$.

almost perfect compensation. However, the discontinuity in the control signal causes large jumps in the response which can be explained as follows. The phaser has a constant magnitude, yet, the switching from one phase angle to another affects the response. The out-

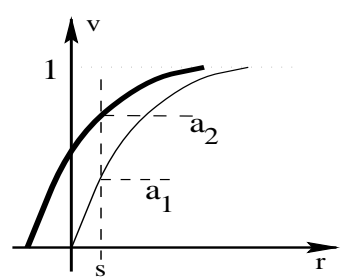

a)

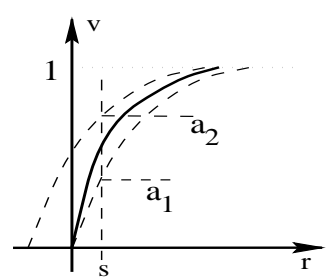

b)
Figure 10: a) Two possible outputs for the variable phaser, thick graph: when $\phi=\phi_{1}$, thin line: $\phi=\phi_{2}$. b) Output of a variable phaser for continuous changes of $\phi$.

put of the variable phaser is different whether using $\phi_{1}$ or $\phi_{2}$ (Figure 10.a)). Furthermore, if a switching has to be done at $r=s$, the phaser will switch from the value $a_{1}$ to the value $a_{2}$ producing the jumps seen in Figure 9. The final version of the compensator changes the compensation continuously with the input signal.

A continuous approximation to Figure 6, can be found using the following equation:

$$
\phi(r(t))=\left\{\begin{array}{c}
-A \tanh (B r(t)-C)-D \\
\text { if }|r(t)| \geq 0 \\
A \tanh (B r(t)+C)-D \\
\text { if }|r(t)|<0
\end{array}\right.
$$




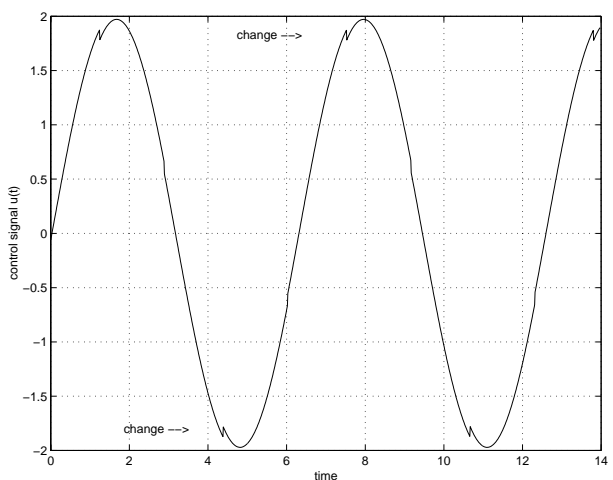

Figure 11: Control signal $u(t)$.

where parameters $A, B, C$, and $D$ are chosen to approximate the discrete function. The continuous variation of the angle as a function of the input for the present problem has the form:

$$
\phi(r(t))=\left\{\begin{array}{c}
-1.8 \tanh (2.75 r(t)-4.8)-0.5 \\
\text { if }|r(t)| \geq 0 \\
1.8 \tanh (2.75 r(t)+4.8)-0.5 \\
\text { if }|r(t)|<0
\end{array}\right.
$$

The discrete and continuous variations of the angle $\phi$ are shown in Figure 12.

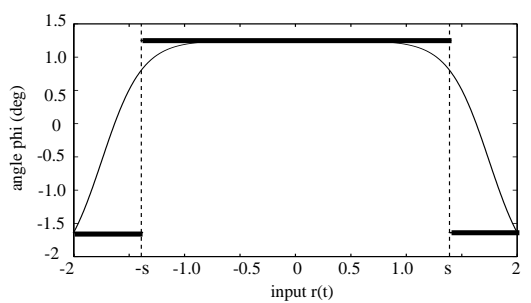

Figure 12: Variation of the angle $\phi$ with the input. Thick line discrete variations, continuous line continuous variations.

Using an angle $\phi$ with continuous variations, Figure 13 is obtained. where the hysteresis has been reduced to a singular nonlinearity.

Experimental Results. An SMA actuator ${ }^{2}$ test bed was built to validate the results for the reduction of hysteresis with saturation obtained above. Figure 14 presents the hysteresis loop of the SMA actuator. This loop has saturation and is not symmetric as the one considered above.

After using the first phaser Figure 15 was obtained. The original loop was divided in just two and not three loops as predicted. This is because the asymmetry of the original loop.

\footnotetext{
${ }^{2}$ A current input to displacement output configuration was used. For technical specifications of the SMA actuator see [6]
}

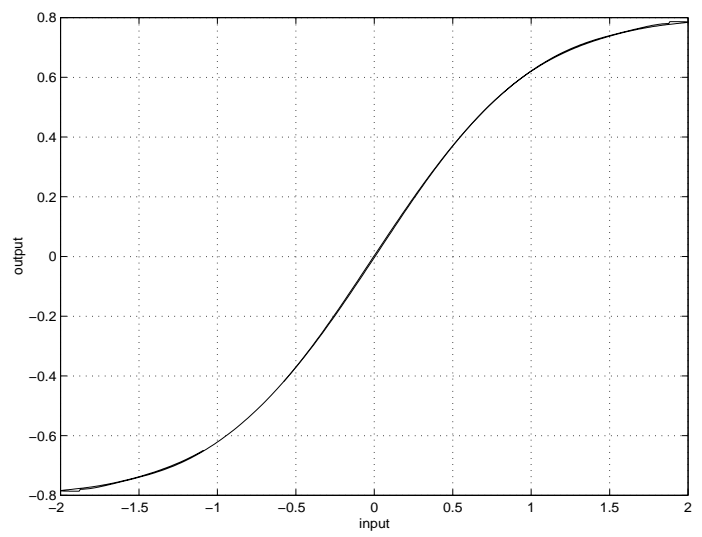

Figure 13: Input-output response after a two stage controller (phasers) was used. The second stage is a phaser varying with continuous values of $\phi$.

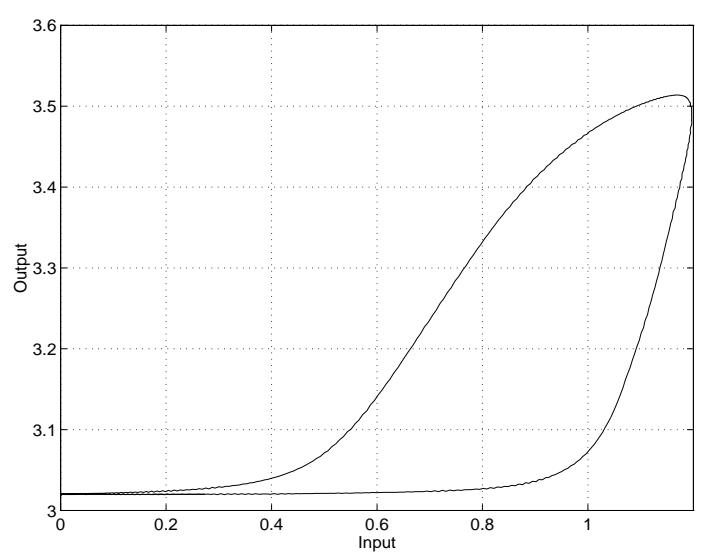

Figure 14: Hysteresis loop of the SMA actuator. Input frequency $0.05 \mathrm{~Hz}$.

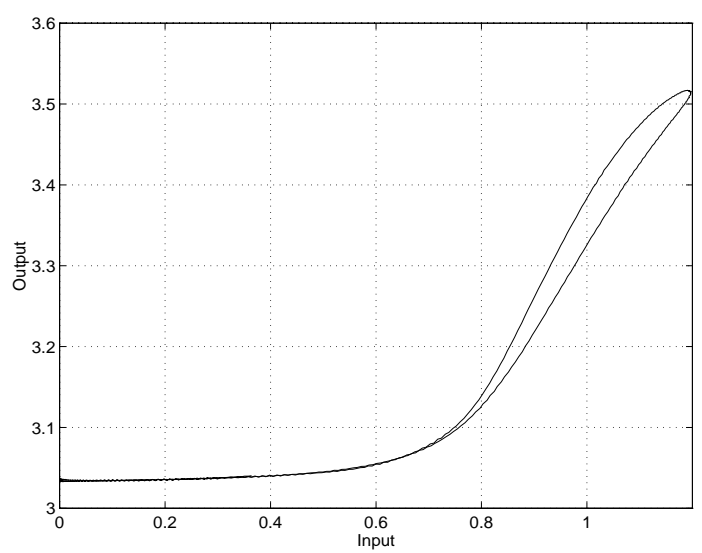

Figure 15: Hysteresis loop of the SMA actuator in series with a phaser with parameter $\phi=24$.

Finally a variable phaser was constructed using the values, $A=3, B=3, C=3$ and $D=3$, and Figure 16 was obtained. 


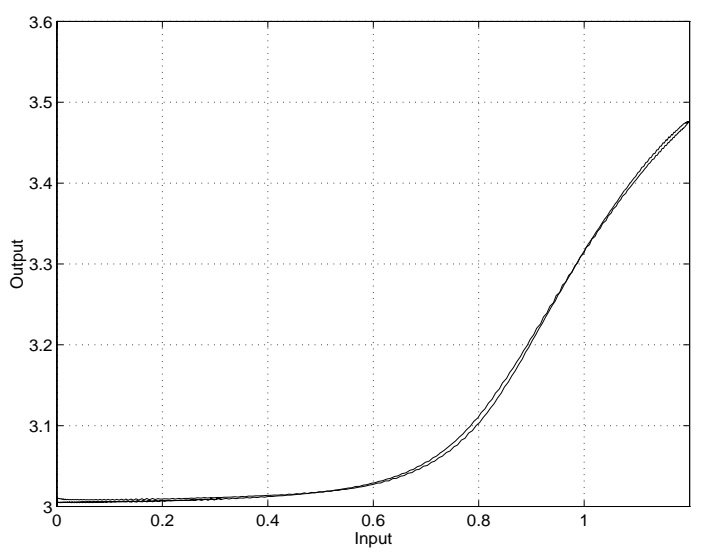

Figure 16: Loop obtained after using two phasers with the SMA actuator.

\section{Conclusions and future work}

A control design strategy by successive approximations has been introduced to reduce the hysteresis commonly found in nonlinear systems, specifically smart materials. The approach uses the so-called phaser, an operator that shifts its periodic input signal by a constant phase angle $\phi$. The phaser when used to reduce the hysteresis without saturation, results is a perfect linear input-output relation. A two stage compensator has been shown in simulation to be able to reduce almost completely the hysteresis with saturation modelled by the hystery unit, and the hysteresis found in SMA actuators.

Current work is carried out toward the development of a complete control design methodology which includes:

- Quantification of performance;

- Ability to handle minor loops;

- Inclusion of the dynamic behavior of the system under consideration.

\section{Acknowledgements}

This research was funded by an operating grant from NSERC, the National Science and Engineering Council of Canada and by IRIS (Phase II), the Institute for Robotics and Intelligent Systems part of Canada's Centres of Excellence Program (NCE), project "MSA-4".

\section{References}

[1] M. Brokate. Hysteresis and phase transitions. New York : Springer, 1996.

[2] J.M. Cruz-Hernández and V. Hayward. On the linear compensation of hysteresis. In Conference on Decision and Control, 1997.
[3] P. Ge and M. Jouaneh. Tracking control of a piezoceramic actuator. IEEE Transactions on Control Systems Technology, 4(3):209-216, May 1996.

[4] M. Goldfarb and N. Celanovic. Behavioral implications of piezoelectric stack actuators for control of micromanipulation. In Proceedings of the IEEE International Conference on Robotics and Automation, pages 226-231, 1996.

[5] R.B. Gorbet and D.W.L. Wang. General stability criteria for a shape memory alloy position control system. In Proceedings of the 1995 IEEE International Conference on Robotics and Automation, volume 3, pages 2313-2319, 1995.

[6] D. Grant and V. Hayward. Variable structure control of shape memory alloy actuators. IEEE Systems and Control Magazine, 17(3):8088, 1997.

[7] D. Hughes and T.J. Wen. Preisach modeling and compensation for smart material hysteresis. SPIE, Active Materials and Smart Structures, 2427:50-64, 1995.

[8] D. Hughes and T.J. Wen. Preisach modeling of a piezoceramic and shape memory alloy hysteresis. IEEE Conference on Control Applications, pages 1086-1091, 1995.

[9] M. Krasnosel'skiǔ and A. Pokrovskiu. Systems with Hysteresis. Berlin; New York: SpringerVerlag, 1989.

[10] I.D. Mayergoyz. Mathematical Models of Hysteresis. Springer-Verlag, 1991.

[11] G. Tao and V. Kokotović. Adaptive control of plants with unknown hysteresis. IEEE Transactions on Automatic Control, 40(2):200-212, February 1995.

[12] M.D. Tom and M.F. Tenorio. The hystery unit -a short term memory model for computational neurons. Technical Report TR-EE-90-63, Purdue University, School of Electrical Engineering, December 1990.

[13] A. Visintin. Differential Models of Hysteresis. Springer-Verlag, 1991. 